\title{
Poststreptococcal Glomerulonephritis in a Patient with Essential Thrombocytosis
}

\author{
Sukru Ulusoy ${ }^{1}$, Gulsum Ozkan², Mehmet Sonmez ${ }^{3}$, Sevdegül Mungan", \\ Rahman Köseoğlu ${ }^{5}$, Muammer Cansız ${ }^{1}$ and Kübra Kaynar ${ }^{1}$ \\ ${ }^{1}$ Department of Nephrology, School of Medicine, Karadeniz Technical University, Turkey \\ ${ }^{2}$ Department of Nephrology, Hatay Antakya State Hospital, Turkey \\ ${ }^{3}$ Department of Hematology, School of Medicine, Karadeniz Technical University, Turkey \\ ${ }^{4}$ Department of Patholology, School of Medicine, Karadeniz Technical University, Turkey \\ ${ }^{5}$ Department of Internal Medicine, School of Medicine, Karadeniz Technical University, Turkey
}

\begin{abstract}
In addition to being the main cause of glomerulonephritis in children, poststreptococcal glomerulonephritis (PSGN) has recently been shown in older patients, especially those with malignancy or diabetes mellitus. The pathogenesis of PSGN has been ascribed to activation of complement 3 (C3) of the alternative complement cascade which, along with immunoglobulin (Ig) G and IgM deposits, is observed in renal tissue. Our aim here is to discuss the probable causes of PSGN developing with isolated IgM deposition in a 52-year-old patient with essential thrombocytosis followed-up over the previous 3.5 years. These characteristics make our case the first to be reported in the literature.
\end{abstract}

(J Nippon Med Sch 2015; 82: 59-63)

Key words: essential thrombocytosis, glomerulonephritis, myeloproliferative neoplasm, poststreptococcal glomerulonephritis

\section{Introduction}

Poststreptococcal glomerulonephritis (PSGN) is a type of glomerulonephritis that develops following streptococcus-related throat and skin infections ${ }^{1}$. It affects children in countries with low levels of socioeconomic development $^{2}$. In Europe and North America PSGN has begun being seen frequently in persons older than 60 years with diabetes mellitus and malignancies, users of intravenous drugs, and alcoholics. ${ }^{3}$.

Myeloproliferative neoplasm (MPN) is a hematological disease characterized by clonal proliferation of granulocytic, megakaryocytic, or erythroid series progenitor cells. Essential thrombocytosis (ET) is a type of MPN ${ }^{4}$. Various renal diseases have been reported in patients with ET and include obstruction of the urinary tract by blood clots, bilateral acute kidney failure related to renal artery thrombosis, and various glomerular diseases ${ }^{5-7}$. However, the pathogenesis of renal diseases in both ET and other MPNs is still unclear.
Our aim in this case report was to report, for the first time in the literature, PSGN in a patient with ET, which is known to accompany various renal diseases.

\section{Case History}

At a hospital where he had presented with hemoptysis 3.5 years previously while under observation for hypertension for 8 years and coronary artery disease for 4 years, a 52-year-old man was found to have a platelet count of $1,600 \times 10^{3} /\left(\times 10^{3} / \mu \mathrm{L}\right)$. Tests for JAK2 and bcr-abl in both specimens of peripheral blood and bone marrow aspirate were negative. Examination of the bone marrow showed increased cellularity, an increase in the number of megakaryocytes, clustering, abnormal localization, and dysplastic changes. The morphologies of both granulocytic and erythrocytic series were normal. On the basis of these findings ET was diagnosed, and treatment was started with hydroxyurea, $1 \mathrm{~g}$ in the morning and $500 \mathrm{mg}$ in the evening. After a follow-up examination, treatment

Correspondence to Sukru Ulusoy, MD, Department of Nephrology School of Medicine, Karadeniz Technical University, 61080

Trabzon, Turkey

E-mail: sulusoy2002@yahoo.com

Journal Website (http://www.nms.ac.jp/jnms/) 
Table 1 Biochemical and hematological variables in the diagnosis and follow-up of the patient

\begin{tabular}{|c|c|c|c|c|c|c|c|}
\hline & $\begin{array}{l}\text { At diagnosis of } \\
\text { essential } \\
\text { thrombocytosis }\end{array}$ & $\begin{array}{l}\text { Second } \\
\text { visit }\end{array}$ & $\begin{array}{l}\text { At the } \\
\text { time of } \\
\text { throat } \\
\text { infection }\end{array}$ & Control & $\begin{array}{l}\text { At } \\
\text { renal } \\
\text { biopsy }\end{array}$ & $\begin{array}{c}\text { First } \\
\text { control } \\
\text { after renal } \\
\text { biopsy }\end{array}$ & $\begin{array}{l}\text { Last } \\
\text { control }\end{array}$ \\
\hline Glucose (mg/dL) & 105 & 98 & 85 & 98 & 104 & 83 & 107 \\
\hline Blood urea nitrogen $(\mathrm{mg} / \mathrm{dL})$ & 21 & 20 & 37 & 30 & 33.3 & 20.6 & 7 \\
\hline Creatinine (mg/dL) & 0.96 & 0.84 & 1.23 & 2.03 & 2.5 & 1.26 & 0.84 \\
\hline Sodium (mmol/L) & 144 & 140 & 138 & 145 & 138 & 143 & 140 \\
\hline Potassium (mmol/L) & 5.3 & 4.8 & 4.8 & 5.5 & 4.4 & 4.3 & 5 \\
\hline Calcium (mg/dL) & 10.1 & 10 & 9.6 & 9.85 & 9.53 & 8.87 & 9.7 \\
\hline Phosphorus (mg/dL) & 3.5 & 3.8 & 3.9 & 3.75 & 4.07 & 4.09 & 2.5 \\
\hline Uric acid (mg/dL) & 6.3 & 6.1 & 5.8 & 5.5 & 5.6 & 4.3 & 6.8 \\
\hline Lactate dehyrogenase (U/L) & 355 & 300 & 523 & 403 & 534 & 600 & 377 \\
\hline Total protein $(\mathrm{g} / \mathrm{dL})$ & 8.1 & 7.8 & 7.9 & 7.8 & 7.61 & 5.64 & 6.9 \\
\hline Albumin (g/dL) & 4.8 & 4.6 & 3.46 & 4.22 & 3.85 & 3.65 & 4.1 \\
\hline Total cholesterol (mg/dL) & & & & 148 & & & \\
\hline Triglycerides (mg/dL) & & & & 250 & & & \\
\hline Low-density lipoprotein (mg/dL) & & & & 86 & & & \\
\hline High-density lipoprotein (mg/dL) & & & & 38.8 & & & \\
\hline Hemoglobin (g/dL) & 15.2 & 14.8 & 9.8 & 11.4 & 11.3 & 11.2 & 13 \\
\hline Hematocrit (\%) & 45.8 & 43.8 & 33.8 & 33.8 & 33.6 & 32.9 & 37.1 \\
\hline White blood cells (U/L) & 6,300 & 6,800 & 2,560 & 4,700 & 5,800 & 11,500 & 8,100 \\
\hline Platelets $\left(\times 10^{3} / \mu \mathrm{L}\right)$ & 1,600 & 450 & 367 & 453 & 450 & 424 & 440 \\
\hline high-sensitivity C-reactive protein (mg/dL) & & & 0.96 & 0.48 & 0.35 & 0.33 & 0.6 \\
\hline Urinalysis density & & 1,018 & 1,020 & 1,020 & 1,011 & 1,014 & 1,020 \\
\hline Protein $(\mathrm{mg} / \mathrm{dL})$ & & - & ++ & - & 300 & 100 & - \\
\hline Erythrocytes & & - & +++ & $10-12$ & 65 & 31 & - \\
\hline Antistreptolysin O (IU) & & & & 13 & 961.67 & & \\
\hline
\end{tabular}

was started with allopurinol, $300 \mathrm{mg} /$ day. Hematological and biochemical values at follow-up are shown in Table 1.

At the hospital where he had presented 3 weeks earlier with a fever of $38.5^{\circ} \mathrm{C}$, sore throat, and nausea, the patient was given an antipyretic and an antibiotic drug whose name he did not know and was then sent home. Laboratory values at that time and at the hospital where he presented several times subsequently are shown in Table 1 . The patient presented to the hematology clinic 1 week previously and was referred to the nephrology department because of a disorder of kidney function. There were no abnormal findings at physical examination at presentation to the nephrology clinic except for splenomegaly. The blood urea nitrogen level was $32.9 \mathrm{mg} / \mathrm{dL}$, and the creatinine level was $2.5 \mathrm{mg} / \mathrm{dL}$. Microscopic examination of the urine revealed dysmorphic erythrocytes and red blood cell casts. Abdominal ultrasonography showed a spleen size of $145 \mathrm{~mm}$ and normal kidney dimensions. Analysis of a 24-hour urine specimen revealed $2.465 \mathrm{~g}$ protein and $1.88 \mathrm{~g}$ albumin. The antistreptolysin O (ASO) level was 961.67 IU. C3 and C4 were normal, and antinuclear antibodies (ANA), Anti-double-stranded
DNA, perinuclear and cytoplasmic antineutrophil cytoplasmic antibody were negative determined. Renal biopsy revealed diffuse mesangial proliferation, neutrophil infiltration, and capillary lumen occlusion (Fig. 1, 2). Immunofluorescence studies revealed granular capillary walls and mesangial deposits of immunoglobulin (Ig) M (Fig. 3). Electron microscopic examination revealed electron-dense deposits in the subepithelial region within the basal membrane (Fig. 4). On the basis of these findings, PSGN was suspected.

Treatment with methylprednisolone was started at a dosage of $1 \mathrm{mg} / \mathrm{kg}$ per day. We planned to continue treatment at this dosage for 1 month, to reduce the dosage, and then to stop treatment after 6 months. After 1 month of treatment, creatinine levels were close to normal. At follow-up after 6 months of treatment, renal function had returned to normal, and the microscopic hematuria and proteinuria had resolved.

\section{Discussion}

Edema developing in association with gross hematuria darkening from red to brown and a decrease in volume is one of the oldest clinical observations in nephrology ${ }^{8}$. 


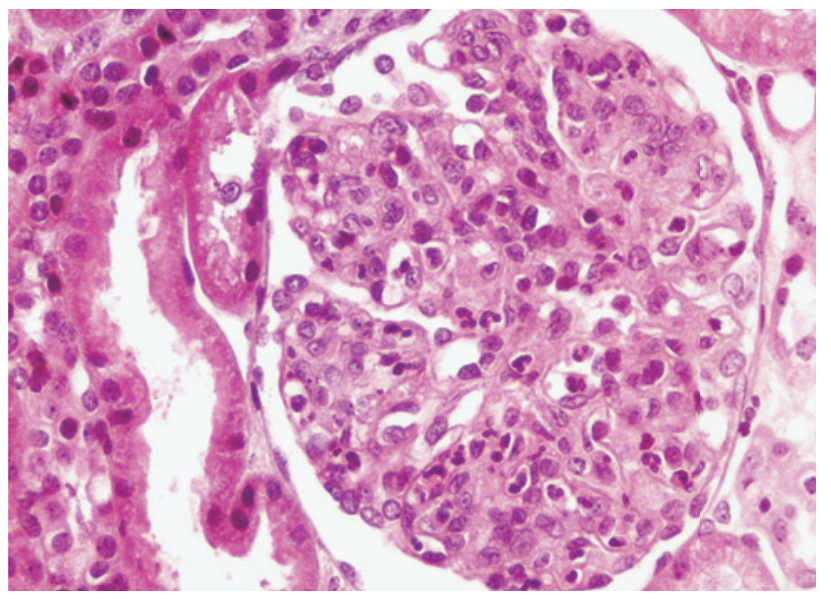

Fig. 1 A representative image of a hematoxylin and eosin-stained section showing diffuse and global endocapillary proliferative glomerulonephritis. The glomerular capillaries are globally occluded by numerous infiltrating neutrophils, and glomerular endothelial and mesangial cells have proliferated (hematoxylin and eosin $\times 400$ )

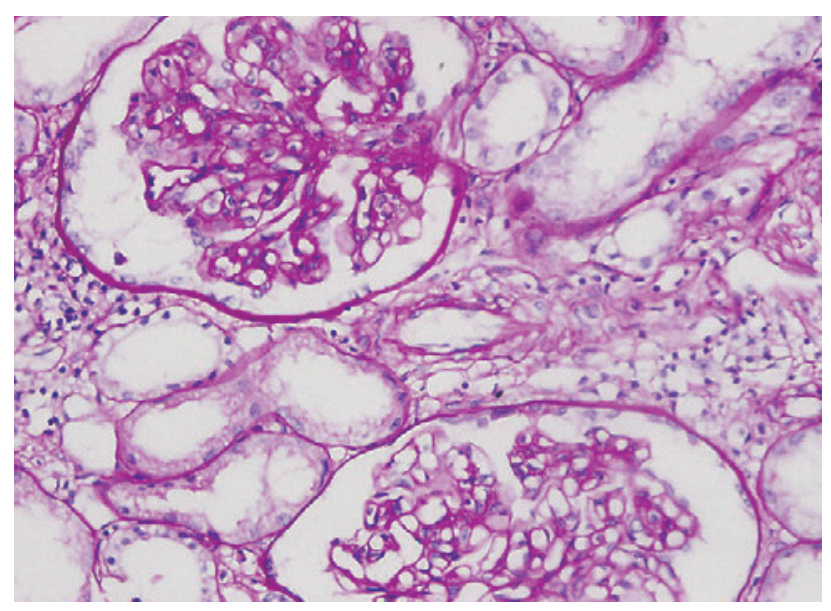

Fig. 2 A representative image of a periodic acid-Schiffstained section showing diffuse and global endocapillary proliferative glomerulonephritis.

This clinical condition was subsequently named acute PSGN, and for years it represented a main cause of nephritis in children². Recently, PSGN has also been observed in adults, in whom it may follow a more severe course. Diabetes mellitus, alcoholism, intravenous drug use, and malignancy are frequently observed in patients in whom PSGN develops at an elderly individuals ${ }^{3}$. Our patient was suspected to have PSGN because of a history of throat infection and the presence of elevated levels of ASO and proliferative glomerulonephritis. We believe that the presence of ET, a type of MPN, in our 52-yearold patient facilitated the development of PSGN.

Factors that have been proposed in the pathogenesis of

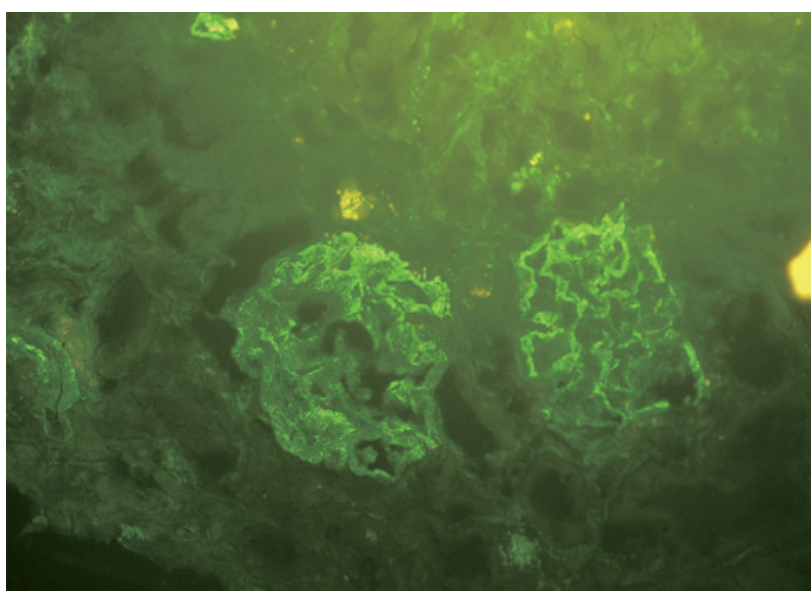

Fig. 3 A representative immunofluorescence image showing coarsely granular positivity (starry sky pattern) in a global glomerular capillary wall and mesangial distribution $(\operatorname{anti-IgM} \times 400)$.

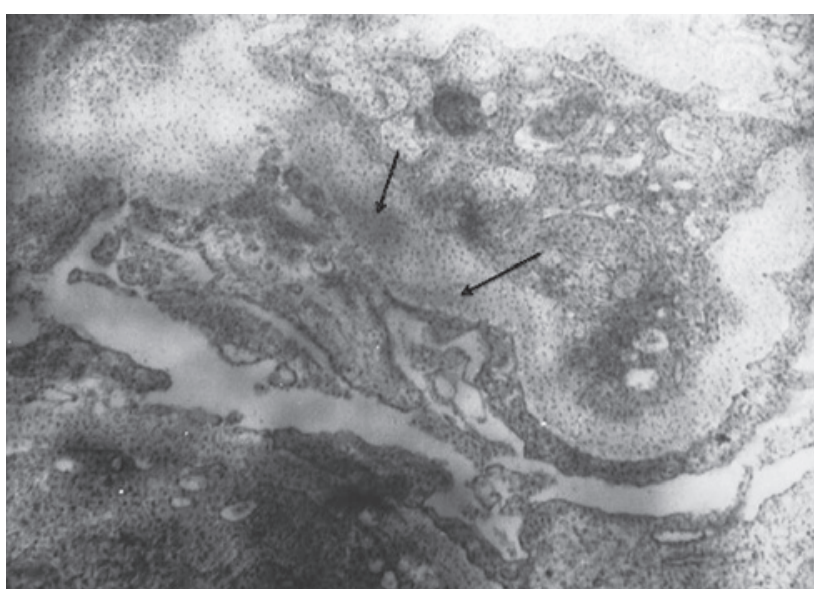

Fig. 4 A representative electron micrograph showing subepithelial electron-dense deposits (arrows) in the basement membrane.

PSGN include C3 nephritogenic factor, and activation of the alternative complement pathway (C3, C4, properdin) via streptococcal component ${ }^{9}$. Therefore, a low serum C3 level and immunofluorescent staining for C3 in renal tissue are seen in most patients with PSGN. Compleman levels usually return to normal within 6 weeks from the onset of glomerular disease ${ }^{1}$. However, as shown by Blyth et al., the duration of low complement levels is variable, and there may be no correlation between creatinine and complement levels ${ }^{10}$. We attributed the normal C3 level in the present case to the patient seeking care approximately 4 weeks after throat infection. Had he come to the nephrology clinic earlier, we believe we would have been able to document a low C3 level. Isolated C3 staining may be seen in many cases, and immunofluorescent staining accompanied by $\operatorname{IgG}$ and $\operatorname{IgM}$ 
may also be observed ${ }^{11}$. However, there are, to our knowledge, no previous reports of a case of PSGN with isolated IgM deposition. Our patient had no findings suggestive of Waldenstrom's macroglobulinemia at bone marrow biopsy performed due to ET. We believe ours is the first such case.

While PSGN in children may cause acute renal failure in patients with crescent formation, it generally follows a benign course, and complete remission is usually observed $^{12}$. Factors affecting the course and chronicity of PSGN include age, levels of creatinine and albumin, low levels of C3 at time of renal biopsy, and the presence of endocapillary proliferation and interstitial inflammation $^{3,11}$. Restriction of fluid and sodium intake and the use of diuretics and antihypertensive agents are generally required, together with hemodialysis in some patients. However, steroids and immunosuppressive agents have been administered to patients in whom clinical and laboratory markers indicated a poor prognosis, and a better remission rate was obtained ${ }^{12}$. Because of our patient's age (52 years), the high creatinine level, and the presence of endocapillary proliferation, treatment with methylprednisolone was started at a dosage of $1 \mathrm{mg} / \mathrm{kg}$ per day. After 1 month of treatment at this dosage, we planned to reduce the dosage and then to stop treatment after 6 months.

The MPNs are a group of clonal hematopoietic stem cell diseases ${ }^{13}$ that include chronic myelogenous leukemia, polycythemia vera, ET, primary myelofibrosis, systemic mastocytosis, chronic eosinophilic leukemia not otherwise specified, and chronic neutrophilic leukemia. In patients with MPNs, disorders of renal function may be observed, albeit rarely, for various reasons. These reasons are restricted to case reports and small case series in the literature ${ }^{4}$. There have been rare case reports of disorders of renal function in patients with ET. As expected, disorders of renal function have been reported as acute renal failure associated with renal artery and vein thrombosis and obstruction of the urinary tract by blood clots caused by thrombotic complications and have also, rarely, been reported in association with focal segmental glomerulosclerosis and fibrillary glomerulonephritis of uncertain etiopathology $y^{5-7,14}$. To our knowledge, the present case is the first to be reported of ET accompanying PSGN. The facilitating factor in the development of PSGN in our patient may have been leukopenia developing in association with hydroxyurea use at the time of throat infection.

Hydroxyurea is a widely used antimetabolite antican- cer drug. It reduces the synthesis of deoxyribonucleotides through the inhibition of ribonuclease reductase ${ }^{15}$. Hydroxyurea can be used safely to treat patients with ET. The most frequently seen side effects are pneumonitis and skin ulcers, while leukopenia is also observed, as with several other anticancer drugs ${ }^{16}$. Hydroxyurea has rarely been reported to impair renal function; this effect may be associated with tumor lysis syndrome and can lead to thrombotic microangiopathy ${ }^{17,18}$. In the present case, the absence of such laboratory findings as hyperuricemia, hyperkalemia, and hyperphosphatemia ruled out tumor lysis syndrome-related kidney injury. Furthermore, histopathological examination ruled out thrombotic microangiopathy-related kidney injury. In light of our patient's history of throat infection, elevated levels of ASO, and histopathological findings supporting PSGN, we suspect the kidney injury in our patient was due to PSGN facilitated by leukopenia developing as the result of hydroxyurea use.

Another possible cause of impaired renal function in our patient is allopurinol. Allopurinol is an analog of hypoxanthine and is used to treat hyperuricemia. The most important side effect limiting its use is drug rash with eosinophilia and systemic symptoms syndrome, which affects the skin, kidneys, and liver ${ }^{19}$. However, because our patient did not have eosinophilia, systemic symptoms, or interstitial nephritis, we did not suspect renal involvement associated with allopurinol use.

In conclusion, the present case is, to our knowledge, the first to be reported of ET accompanied by PSGN and isolated IgM deposition. Several risk factors for the development of PSGN may be present, we recommend early antibiotic therapy and the monitoring of renal function. Also, we believe that monitoring leukocyte numbers is important in patients with ET treated with hydroxyurea and that greater care must be taken when infectious findings are present.

Conflict of Interest: We report no conflict of interest.

\section{References}

1. Kambham N: Postinfectious glomerulonephritis. Adv Anat Pathol 2012; 19: 338-347.

2. Nast CC: Infection-related glomerulonephritis: changing demographics and outcomes. Adv Chronic Kidney Dis 2012; 19: 68-75.

3. Nasr SH, Fidler ME, Valeri AM, et al.: Postinfectious glomerulonephritis in the elderly. J Am Soc Nephrol 2011; 22: 187-195.

4. Said SM, Leung N, Sethi S, et al.: Myeloproliferative neoplasms cause glomerulopathy. Kidney Int 2011; 80: 753- 
759.

5. Bello Nicolau I, Conde Zurita JM, Barrientos Guzman A, et al.: Essential thrombocytosis with acute renal failure due to bilateral thrombosis of the renal arteries and veins. Nephron 1982; 32: 73-74.

6. Votaw ML, Spannuth C Jr, Krish G, Guarderas J: Acute renal failure in a patient with essential thrombocythemia, diabetes mellitus, and heterozygous hemoglobin $\mathrm{C}$ disease. South Med J 1990; 83: 57-59.

7. Au WY, Chan KW, Lui SL, Lam CC, Kwong YL: Focal segmental glomerulosclerosis and mesangial sclerosis associated with myeloproliferative disorders. Am J Kidney Dis 1999; 34: 889-893.

8. Rodriguez-Iturbe B, Musser JM: The current state of poststreptococcal glomerulonephritis. J Am Soc Nephrol 2008; 19: $1855-1864$.

9. Vernon KA, Goicoechea de, Jorge E, Hall AE, et al.: Acute presentation and persistent glomerulonephritis following streptococcal infection in a patient with heterozygous complement factor H-related protein 5 deficiency. Am J Kidney Dis 2012; 60: 121-125.

10. Blyth CC, Robertson PW, Rosenberg AR: Poststreptococcal glomerulonephritis in Sydney: a 16-year retrospective review. J Paediatr Child Health 2007; 43: 446450.

11. Nasr SH, Markowitz GS, Stokes MB, Said SM, Valeri AM, D'Agati VD: Acute postinfectious glomerulonephritis in the modern era: experience with 86 adults and review of the literature. Medicine (Baltimore) 2008; 87: 21-32.

12. Ahn SY, Ingulli E: Acute poststreptococcal glomeru- lonephritis: an update. Curr Opin Pediatr 2008; 20: 157162.

13. Campo E, Swerdlow SH, Harris NL, Pileri S, Stein H, Jaffe ES: The 2008 WHO classification of lymphoid neoplasms and beyond: evolving concepts and practical applications. Blood 2011; 117: 5019-5032.

14. Asaba K, Tojo A, Onozato ML, et al.: Fibrillary glomerulonephritis associated with essential thrombocytosis. Clin Exp Nephrol 2003; 7: 296-300.

15. Saban N, Bujak M: Hydroxyurea and hydroxamic acid derivatives as antitumor drugs. Cancer Chemother Pharmacol 2009; 64: 213-221.

16. Antonioli E, Guglielmelli P, Pieri L, et al.: AGIMM Investigators; Hydroxyurea-related toxicity in 3,411 patients with Ph'-negative MPN. Am J Hematol 2012; 87: 552-554.

17. Chen SW, Hwang WS, Tsao CJ, Liu HS, Huang GC: Hydroxyurea and splenic irradiation-induced tumour lysis syndrome: a case report and review of the literature. J Clin Pharm Ther 2005; 30: 623-625.

18. Shammas F, Meyer P, Heikkila R, et al.: Thrombotic microangiopathy in a patient with chronic myelogenous leukemia on hydroxyurea. Acta Haematol 1997; 97: 184-186.

19. Markel A: Allopurinol-induced DRESS syndrome. Isr Med Assoc J 2005; 7: 656-660.

(Received, April 9, 2014)

(Accepted, August 20, 2014) 